\title{
LOS CLUBS DE SUSCRIPTORES COMO NUEVO MODELO DE FINANCIACIÓN DE LA PRENSA ESPAÑOLA
}

\author{
Subscribers' clubs: A new funding model for the \\ Spanish press
}

José-Vicente García-Santamaría, María-José Pérez-Serrano y Lidia MaestroEspínola

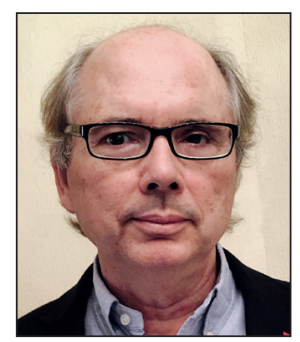

José-Vicente García-Santamaría es licenciado en periodismo y comunicación audiovisual por la Universidad Complutense de Madrid, doctor en ciencias de la comunicación por la Universidad Rey Juan Carlos y ha realizado estudios de dirección de empresa en el IESE. Ha ejercido una dilatada labor profesional como consultor de comunicación y marketing para empresas del lbex y trabajado para el ICO y BBVA. Es profesor en el Departamento de Periodismo y Comunicación Audiovisual de la Universidad Carlos III de Madrid y director del grupo de investigación del Instituto para la Innovación Periodística (2IP). http://orcid.org/0000-0003-4659-3975

Universidad Carlos III de Madrid, Facultad de Humanidades, Comunicación y Documentación C/ Madrid, 126. 28903 Getafe, Madrid, España jvgsanta@hum.uc3m.es

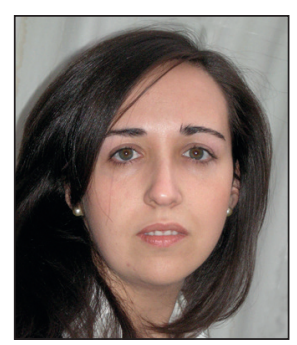

María-José Pérez-Serrano es licenciada en periodismo y doctora en ciencias de la información por la Universidad Complutense de Madrid (UCM), premio extraordinario de doctorado 2006-2007 y $M B A$ con la especialidad de gestión financiera. Es profesora del Departamento de Periodismo IV (Empresa Informativa) de la UCM. Su trayectoria y producción científica se vinculan al estudio de la empresa informativa y específicamente a la concentración de medios de comunicación y su influencia en el pluralismo.

http://orcid.org/0000-0002-2190-7619

Universidad Complutense de Madrid, Facultad de Ciencias de la Información Avda. Complutense, s/n. 28040 Madrid, España mariajoseperezserrano@pdi.ucm.es

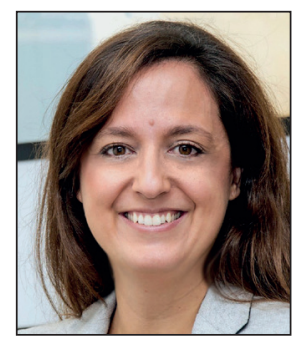

Lidia Maestro-Espínola es doctora en comunicación audiovisual y publicidad por la Universidad Rey Juan Carlos y licenciada en publicidad y relaciones públicas por la Universidad Complutense de Madrid. Es master en Cine, televisión y medios interactivos por la Universidad Rey Juan Carlos y MBA por la EOI. Es profesora del Área de Comunicación y Marketing en grado y master de la Universidad Internacional de La Rioja, y coordinadora académica del Master universitario de publicidad integrada: creación y estrategia.

http://orcid.org/0000-0003-4866-3412

Universidad Internacional de La Rioja, Facultad de Ciencias Jurídicas, Sociales y Humanidades Almansa, 101. 28040 Madrid, España lidia.maestro@unir.net

\section{Resumen}

Hasta el año 2014, y al margen de algunas experiencias poco exitosas, no se apreciaba una corriente muy favorable a la instalación de un pay wall en los grandes diarios españoles. Pero sería a partir de ese mismo año cuando los directivos de los mayores grupos de comunicación españoles se decantasen por desarrollar estrategias de affinity clubs en la senda que ya habían recorrido publicaciones relevantes en Latinoamérica como La nación de Buenos Aires o El mercurio en Santiago de Chile. Como en la mayor parte de los países en los que esta estrategia ha tenido éxito, las principales cabeceras de la prensa, tanto en papel como en digital, pusieron en marcha programas de fidelización de sus lectores, organizando clubs con beneficios exclusivos para que sus socios disfrutasen de una amplia gama de productos y servicios a cambio de una cuota mensual. 


\section{Palabras clave}

Modelos de negocio; Prensa; Periódicos; Financiación social de los media; Clubs de lectores; Clubs de suscriptores; Pago por contenidos; Periodismo digital; Periodismo online; Grupos de comunicación; Comercio electrónico.

\section{Abstract}

Until 2014, and setting aside some unsuccessful experiences, there had not been a successful implementation of a pay wall in a major Spanish newspaper, but beginning in 2014 newspaper executives opted to create affinity clubs following strategies that had already been implemented in similar Latin American newspapers like La nación of Buenos Aires and El mercurio of Santiago de Chile. As in most countries where this strategy has been successful, it addresses the loyalty of its print and digital consumers by organizing clubs and offering exclusive benefits on a wide array of products for a monthly fee.

\section{Keywords}

Media business models; Press; Newspapers; Financing social media; Affinity clubs; Paid content; Journalism online; Communication groups; E-commerce.

García-Santamaría, José-Vicente; Pérez-Serrano, María-José; Maestro-Espínola, Lidia (2016). “Los clubs de suscriptores como nuevo modelo de financiación de la prensa española”. El profesional de la información, v. 25, n.3, pp. 395-403.

http://dx.doi.org/10.3145/epi.2016.may.09

\section{Introducción y planteamiento teórico}

La discusión sobre los modelos de financiación de la prensa en el entorno digital es un debate recurrente en el terreno académico y profesional. Desde la aparición de las ediciones digitales se ha producido un elevado grado de experimentación sobre los modelos a adoptar:

- publicidad;

- pago vía suscripción o venta por unidad;

- modelo combinado: edición digital más un plus;

- modelo de comercio electrónico: comisión en función de los clics de los usuarios.

Sin embargo, no existe un único modelo de negocio para el periodismo digital (Herbert; Thurman, 2007), a pesar de las preferencias de algunos estudiosos por:

- micropagos (Sirkkunen; Cook, 2012);

- crowdfunding (Pérez-Bahón; Clemente-Fernández; López-Aboal, 2015);

- freemium (Anderson, 2009; Leiva, 2009);

- creación de entidades sin ánimo de lucro para sostenerlos (Cagé, 2016).

Tampoco está claro que la mejor opción sea generar variadas fuentes de ingresos (Bleyen; Van-Hove, 2007; Chyi, 2005) para no depender exclusivamente de una sola.

Desde 2014 todos los grandes diarios españoles han perfilado un modelo de club de suscriptores como alternativa al pay wall tradicional

El verdadero problema es que se ha desestabilizado el modelo económico de los medios de comunicación (Poulet, 2011). Los diarios han sido los más afectados al ser incapaces de encontrar formas rentables en la Red (Salaverría, 2010). La prensa pasó de tener una estrategia defensiva en 1995 a revitalizar quince años más tarde la idea del cobro
(Vara-Miguel, 2014). Según Niles (2010), sólo existen tres modelos que permitan obtener ingresos a los editores: la publicidad, el crowdfunding y las compras directas de sus productos (suscripciones).

En España, desde 2010 se han producido numerosas iniciativas emprendedoras con interesantes proyectos informativos que conforman un variado abanico de opciones (GarcíaSantamaría, 2014). Mientras tanto y tras producirse una "tormenta económica perfecta" para los media, la prensa de papel ha perdido entre 2008 y 2014 un total de 5,4 millones de lectores (AEDE, 2015), aunque su resultado operativo fuese positivo en 30 millones de euros en ese último año. Pero la crisis de la prensa no es sólo económica. Como indica Díaz-Nosty (2013, p. XV), esta crisis es "en realidad, el fruto de la inadaptación tecnológica de una industria vinculada a un soporte informativo y publicitario de altos rendimientos económicos", que además se ha mostrado incapaz de liderar las oportunidades que ofrecía internet.

\section{La estrategia seguida por las empresas periodísticas es un modelo de fideliza- ción de clientes propio de casi todas las industrias business-to-consumer (B2C)}

De ahí que la refundación de los modelos de negocio de la prensa y su adaptación a la convergencia digital se haya convertido en una necesidad imperiosa (Casero-Ripollés, 2010). Y su búsqueda ha introducido importantes cambios en la dirección de los diarios de referencia españoles y sus grupos de comunicación. La gran novedad en España es que después de un período de tiempo en el que no había ninguna corriente favorable a instalar sistemas de suscripción (pay walls o "muros de pago"), se observa cómo a partir de 2014 la mayoría de grandes diarios han adoptado estrategias de affinity clubs (clubs de suscriptores o clubs de lectores). En este modelo, los suscriptores (o los lectores en el caso latinoamericano) de- 
ben satisfacer una cuota mensual para tener acceso no sólo a los contenidos del diario sino también a los privilegios del club. Es el caso entre otros de Reforma (México), El comercio (Perú), La prensa (Honduras), La nación (Argentina), El mercurio (Chile) o La tercera (Chile).

http://www.reforma.com

http://elcomercio.pe

http://www.laprensa.hn

http://www.lanacion.com.ar

http://www.elmercurio.cl

http://www.latercera.com

Entre los diarios "nativos digitales" españoles, una parte importante continúa apostando por la publicidad como medio seguro para financiarse y solamente unos pocos se decantan por métodos mixtos (freemium), por novedosas fórmulas de crowdfunding o por la puesta en marcha de alianzas estratégicas. Al margen del éxito económico y editorial del caso específico de Elconfidencial.com, existen dos modelos que desean apartarse de la senda publicitaria para salvaguardar su independencia editorial. Únicamente Infolibre y Eldiario.es parecen disponer de un proyecto claro para el cobro de contenidos en la Red, un proyecto al que se ha unido también el club de lectores de La marea. Además se pueden señalar otros modelos:

- la alianza estratégica de La marea con Prisa;

- las fórmulas de crowdfunding de Eldiario.es;

- la combinación exitosa de venta de revistas y suscripciones, caso de Jot Down;

- el triunfo económico de El español. http://www.elespanol.com

\section{Objetivos y metodología}

Un modelo de negocio se relaciona en esencia con la forma en la que un proyecto empresarial logra dinero y entrega valor a los clientes (Palacios-Preciado; Duque-Oliva, 2011). Según Picard (2002), puede ser definido como la arquitectura del producto, sus servicios y flujos de información, e incluye una descripción de sus actividades, beneficios potenciales para los intervinientes y fuentes de ingresos de las que se nutrirán sus presupuestos.

La innovación en el modelo de negocio es un factor crítico para el éxito de un proyecto, sobre todo en entornos complejos y cambiantes (Giesen el al., 2010). Los directivos de las empresas deben ser capaces de innovar en los tres elementos más importantes del diseño de modelo de negocio: contenido, estructura y forma de gestión (Zott; Amit, 2009).

Este artículo examina la irrupción del nuevo modelo de negocio de los "clubs de suscriptores" en España como medio innovador y estable de financiación de la prensa, que promete mejores resultados para los editores que las estrategias tradicionales más centradas en el pago por los contenidos. El objetivo es también comprobar las similitudes y diferencias que nos separan de otros modelos en el ámbito latinoamericano.
La investigación está basada en:

- método Delphi (Astigarraga, 2002) fundamentado en la consulta de expertos con objeto de llegar a opiniones de consenso en grupo;

- análisis de la oferta de clubs de suscriptores existentes en España, y las de los clubs de lectores en los principales países latinoamericanos.

El proceso de trabajo comenzó con el diseño de un cuestionario semiestructurado -en el que intervino un equipo de expertos del Instituto para la Innovación Periodística (2IP)que permitiese después establecer comparaciones y detectar tendencias (García-Santamaría; Clemente-Fernández; López-Aboal, 2013), y estudiar la evolución de las formas de financiación del sector y los cambios organizativos que habían comportado. Se definieron las preguntas clave, pero no sólo se abordaron esos dos asuntos, sino que también se afrontaron otros como arquitectura del producto, servicios, flujos de información, fuentes de ingresos y descripción de beneficios potenciales, es decir, todos aquellos aspectos que proporcionan valor estratégico al medio.

Se escogieron entrevistas en profundidad con el fin de disponer de material de estudio sustentado en el valor atribuible a las respuestas ofrecidas por los responsables de los principales medios (Taylor; Bogdan, 1987; Ruiz-Olabuenaga, 2008). Estas entrevistas se realizaron durante los meses de abril a noviembre de 2015 siguiendo el cronograma de una entrevista cada diez días aproximadamente, que se fue ajustando a las circunstancias espacio-temporales de los participantes. La mayor parte fueron realizadas al máximo responsable del medio (nativos digitales), aunque en los casos de Elconfidencial y Eldiario.es las conversaciones se hicieron con los directores de desarrollo. Y en los diarios de referencia se entrevistó al director general del grupo de co- 
Tabla 1. Medios españoles analizados

\begin{tabular}{|l|l|}
\hline \multicolumn{1}{|c|}{ Medio } & \multicolumn{1}{c|}{ Url } \\
\hline ABC & http://www.abc.es \\
\hline El país & http://elpais.com \\
\hline El mundo & http://www.elmundo.es \\
\hline La razón & http://www.larazon.es \\
\hline La vanguardia & http://www.lavanguardia.com \\
\hline El periódico de Catalunya & http://www.elperiodico.com/es \\
\hline El diario.es & http://www.eldiario.es \\
\hline Infolibre & http://www.infolibre.es \\
\hline El confidencial & http://www.elconfidencial.com \\
\hline La marea & http://www.lamarea.com \\
\hline Es materia & http://esmateria.com \\
\hline Público & http://www.publico.es \\
\hline Voz populi & http://wozpopuli.com/inicio \\
\hline Jot down & http://www.jotdown.es \\
\hline La información & http://www.lainformacion.com \\
\hline
\end{tabular}

municación al que pertenecen estos periódicos, así como a los responsables de los clubs de suscriptores.

La selección cualitativa y representativa se centró en:

- los grandes diarios de referencia (ABC, El país, El mundo, La razón, La vanguardia, El periódico de Catalunya) y los grupos de comunicación a los que pertenecen (Vocento, Prisa, Unidad Editorial, Planeta, Godó y Zeta);

- diarios nativos digitales que representasen adecuadamente los diferentes modelos de financiación que nos interesaba analizar: Eldiario.es, Infolibre, Elconfidencial. com, La marea, Esmateria, Público, Vozpopuli.com, Jot down y Lainformación.com. A este grupo de quince medios españoles (tabla 1) habría que sumar las entrevistas realizadas en Chile y Argentina a responsables de El mercurio y La nación. ta en marcha de estrategias de fidelización para conservar esa base de clientes que les permitiese afrontar con éxito el tránsito hacia el modelo digital. El 23 de mayo de 2014, Unidad Editorial, que parecía estar rezagada y ajena a estas iniciativas, anunció la puesta en marcha de El club y dio la bienvenida a los suscriptores de las principales cabeceras del grupo que comenzarían a formar parte de un exclusivo programa de beneficios, con 1.500 establecimientos adheridos en toda España e importantes descuentos en ocio, compras, restaurantes, hoteles, belleza, salud o motor. En definitiva, un modelo que les acercaba a las estrategias emprendidas desde hacía unos cuantos años por prestigiosos diarios latinoamericanos.

http://www.elclub.es

En el año 2014 todos los grandes diarios españoles ya habían perfilado un modelo de club de suscriptores. Los de carácter más sofisticado se asemejaban a los modelos latinoamericanos, mientras los más convencionales ofrecían de manera regular ofertas de compras a sus lectores e incluso un conjunto de tiendas virtuales. Así, en el diario La razón, su tienda virtual, denominada Comprix La razón, es un club exclusivo donde se pone a disposición de los lectores online productos y planes de ocio acompañados de interesantes descuentos. Y los quioscos digitales como Orbyt o Kiosko y más tienen también su apartado de promociones exclusivas, como aseguran en estos medios.

http://quiosco.orbyt.es

http://www.kioskoymas.com

La estrategia seguida por las empresas periodísticas es similar también a la emprendida desde hace años por las grandes cadenas de comercio minorista, con el diseño de acciones dirigidas al establecimiento y mantenimiento de relaciones intensas y duraderas con los clientes para incrementar su lealtad hacia la marca (Herrero-Crespo; Rodríguez-del-Bosque; Pérez-Ruiz, 2009). Es una táctica orientada a conseguir el beneplácito del consumidor mediante la prestación de servicios de valor añadido. Es decir, no constituyen tanto

\section{Análisis sobre los clubs de \\ suscriptores y la fidelización}

Desde las primeras décadas del siglo XX, el hecho de que un diario pudiera contar con un elevado número de suscriptores constituía, desde el punto de vista de las empresas periodísticas, la mejor garantía de su estabilidad (Rodríguez-Infiesta, 2008). Pero ya en la era de internet, las fallidas experiencias de cobro de El país entre los años 2000 y 2001, y la posibilidad de perder su base de lectores, hicieron desistir a los responsables de los grandes diarios de continuar por esa vía.

Sin embargo, y como hemos apuntado antes, a partir de 2014 y de forma continuada, diarios como El país o grupos de comunicación como Vocento, comenzaron a virar sus grandes plataformas hacia un modelo que cabía interpretar muy próximo al de los clubs de lectores y, más específicamente, a la pues-

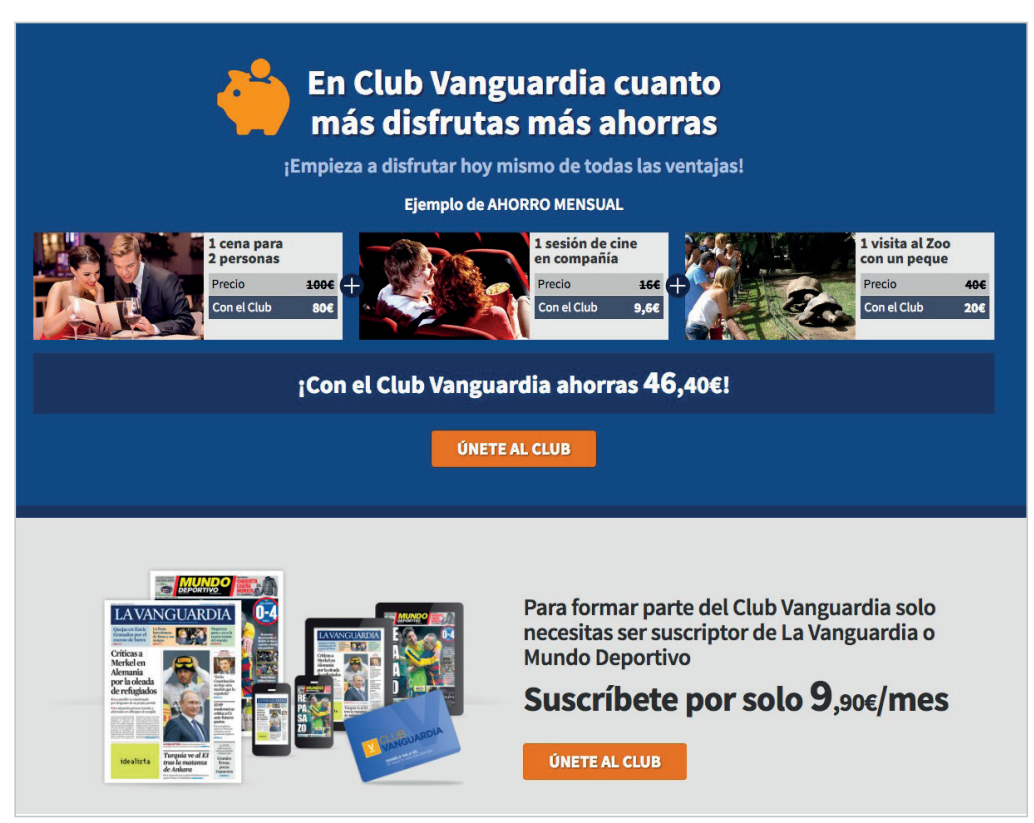

http://club.lavanguardia.com 
herramientas de retención de clientes como programas de fidelización que premian al cliente duradero. Como aseguran en Elconfidencial.com, hunden sus raíces en el marketing relacional, que persigue establecer, mantener y enriquecer las relaciones con clientes y otros asociados para conseguir mayores beneficios (Alfaro-Faus, 2004).

Además se sigue la senda trazada entre 2010 y 2012 por la oferta de cupones de descuento online gracias al modelo de compra colectiva o couponing, que se convirtió en una nueva fuente de ingresos en internet (Gómez-Borrero, 2015). Sitios como LetsBonus (septiembre 2009), Groupon (creado en 2008, en España desde marzo 2010), Offerum (marzo 2010) y Groupalia (mayo 2010) ofrecían volúmenes de negocio interesantes. Iniciativas a las que se unieron entre 2011 y 2013, y con propuestas propias, grupos de comunicación como Prisa (Planeo), Godó (Vivirvip.com) o Vocento (Oferplan).

Asimismo, como apuntaba el director general de Unidad Editorial, José María Cabrerizo, este es un modelo de fidelización de clientes propio de casi todas las industrias B2C (business-to-consumer), pero en el que apenas existe esfuerzo proactivo de venta.

Los perfiles profesionales que se están contratando para desarrollar los clubs de suscriptores están transformando la estructura organizativa de la vieja empresa periodística

Como han destacado en las entrevistas los directivos de los principales grupos mediáticos a los que hemos tenido acceso, la creación de estos clubs supone una sustancial modificación del canal de distribución convencional (punto de venta o quiosco) en busca de una acción voluntaria y sistemática de venta, con acciones muy segmentadas de marketing y el uso de datos con socios que también usan sus propios datos para adecuar sus acciones al público objetivo (targetizar).

El objetivo final es que todos los suscriptores (del papel y digital) acaben siendo miembros del club. Para ello el conocimiento del cliente se convierte en algo esencial. Por lo tanto, los clubs de suscriptores comprenden además tres áreas vitales para controlar el negocio de la prensa:

- targetización de la publicidad;

- acciones proactivas del B2C (business-to-consumer); - nuevas vías de colaboración B2B (business-to-business).

Esas transformaciones conllevan un cambio radical en los perfiles profesionales que deben contratar diarios y grupos de comunicación, que se unen a las figuras emergentes en las redacciones digitales más vanguardistas, que ya se encuentran pobladas de matemáticos, informáticos o realizadores de vídeo, etc. A ellos se une la aportación de nuevos diseñadores web, estadísticos, arquitectos de datos y nuevos perfiles comerciales y de marketing capaces de llevar a cabo acuerdos con empresas para ofrecer en el club una amplia variedad de servicios.

Todos estos perfiles, como apuntan desde Elconfidencial. com, Unidad Editorial, Prisa y Vocento, no sólo están transformando radicalmente el universo redaccional de los diarios sino también la estructura organizativa de la propia empresa periodística, o más bien, de las nuevas empresas digitales. Esta transformación tan radical no sería posible sin la designación previa, en un proceso de apenas tres años, de nuevos responsables editoriales y de estrategia de los principales grupos mediáticos, tal y como hemos podido constatar en nuestra investigación.

\section{Comparación de estrategias entre clubs}

Las categorías de los productos o servicios que estos clubs ofrecen son muy similares, tanto en España como en Latinoamérica, y abarcan desde ofertas gastronómicas de índole muy variada, a promociones turísticas, ofertas de moda y belleza o entradas para museos o espectáculos variados (teatro, cine, ballet, etc.). Las diferencias, aunque pequeñas, las encontramos en la periodicidad de suscripción mínima exigida y en el número de tarjetas disponibles por suscriptor para beneficiarse de las oportunidades del club.

La nación de Buenos Aires lleva trabajando desde 1995 en acciones de marketing destinadas a ofrecer beneficios a sus lectores, y dispone de un club de suscriptores desde 2003. Adopta fórmulas flexibles y permite suscribirse al club -ade- 
más de su categoría premium- durante dos días a la semana o combinar suscripciones de diario con la de conocidas revistas (Rolling stone). http://club.lanacion.com.ar

El diario El mercurio (Chile), otro ejemplo de éxito con más de 250.000 socios, promueve conciertos de música en Santiago de Chile. Otra particularidad de esta cabecera es la creación en octubre de 2012 del Club Verde, un programa de beneficios y descuentos relacionados con un consumo medioambiental responsable.

http://www.clubdelectores.cl

Se puede comprobar la pujanza de ambos diarios analizando su sistema de geolocalización de locales comerciales o recorriendo zonas emblemáticas de Buenos Aires (Palermo) o los principales centros comerciales de Santiago de Chile. Así se comprende la importancia de los beneficios de sus tarjetas de miembros del club en forma de importantes descuentos.

La propuesta de Prisa es El paíst, que no sólo ofrece descuentos, invitaciones y entradas, sino también un mayor acercamiento a los suscriptores con sus jornadas de puertas abiertas para conocer cómo se trabaja en el entorno digital. http://elpais.com/suscripciones/elpaismas.htm/

Los directivos de grupos españoles de comunicación consideran que en el momento actual la estrategia más acertada pasa por la creación de grandes clubs de suscriptores

En Unidad Editorial los beneficios del club comenzaron por El mundo y se han extendido a sus principales publicaciones como Marca, Expansión o Telva. Todas se engloban en $E$ I club, compuesto por 350 marcas y 1.500 establecimientos repartidos por toda España. A través de la aplicación móvil de El club los socios, mediante un sistema de geolocalización, pueden consultar los establecimientos más cercanos. Además han creado El club experiencias donde pueden participar en sorteos y disfrutar de experiencias exclusivas. http://www.elclub.es

También existen otros clubs de suscriptores como los del Diario de Navarra o La vanguardia. El diario del Grupo Godó ofrece hasta seis tarjetas por suscriptor y utiliza una cuenta independiente en Twitter (con 1.700 seguidores, @Club_ Vanguardia) para el programa de beneficios. https://clubsuscriptor.diariodenavarra.es http://club.lavanguardia.com/es

El periódico de Catalunya (Grupo Zeta) llegó a un acuerdo en noviembre de 2014 con el Club de cultura TR3SC para que todos los suscriptores que estén abonados al diario por un período mínimo de tres meses en su edición impresa o un año en su edición digital reciban de forma gratuita el carnet de este club.

http://especiales.elperiodico.com/suscriptores/clubsuscriptor-tr3sc

Las diferencias entre los modelos más evolucionados de Latinoamérica (Argentina, Chile y Perú), y los modelos españoles se centran en:

- excelente base de suscriptores de los diarios latinoamericanos (en algunos casos, por encima de los 200.000), que les permiten conservar la pujanza de la era analógica;

madurez y desarrollo tecnológico de las propuestas de diarios como El comercio, El mercurio, La tercera o La nación. No sólo porque son capaces de convertir en realidad la devolución de cada 6 dólares por cada dólar invertido en la suscripción al club sino por su facilidad de participar activamente en las propuestas de ocio de ciudades como Buenos Aires, Santiago de Chile, Lima o Caracas.

\section{El break-even y los clubs de suscriptores}

Las cifras de suscriptores de los diarios españoles no son una información pública ni fácil de obtener. Además, los anuarios que recogen estas cifras, como sucede en el caso de $A E D E$, tienden cada vez más a categorizar y ofrecer cantidades agrupadas por sectores económicos y no desglosadas por conglomerados mediáticos, lo que dificulta la evaluación de las estrategias seguidas por las empresas periodísticas. Para ayudar a resolver esta cuestión, las actas mensuales que audita OJD (Oficina de Justificación de la Difusión), donde aparecen las suscripciones individuales (ejemplares vendidos y servidos a los suscriptores de la publicación) y las suscripciones colectivas (ejemplares vendidos a patrocinadores y empresas que son servidos a los suscriptores que 
estas seleccionan), podrían contribuir a conocer la situación real del mercado.

Si el objetivo fuese rentabilizar cada suscriptor, lector a lector, y no maquillar las cifras de difusión regalando bloques corporativos o accesos gratuitos a los pay walls digitales, como nos señalan algunos de los entrevistados, las fuentes de ingresos de los diarios serían mucho más claras. En lo que también coinciden otros responsables de medios es que la combinación de un contenido editorial impreso con las ventajas que proporciona la tecnología a la hora de conocer sus audiencias digitales debe proporcionar a los medios de comunicación una oportunidad de recuperar su rentabilidad.

Las diferencias entre los modelos latinoamericanos y españoles son el elevado número de abonados de muchos clubs latinoamericanos y su mayor desarrollo tecnológico

Otro problema es que estos programas de beneficios están delimitados geográficamente a España, con la restricción que eso conlleva. Apuestas como la del diario El país por Latinoamérica, especialmente México, pero también con ediciones en Chile, Argentina o República Dominicana, podrían encontrar una oportunidad de negocio ampliando la oferta de ventajas exclusivas en esos países.

\section{Conclusiones}

Desde el inicio de las ediciones digitales se ha producido un elevado grado de experimentación en las estructuras de ingresos. Pero después de veinte años de ensayos y errores, la conclusión para la industria es que ninguno de ellos ha sido capaz de generar aún la necesaria masa monetaria para hacer viable el modelo actual de periodismo online. Aunque la cuestión, como apunta Varela (2011), "no es pago o gratis. El problema es cómo se diseña un negocio de pago y cómo se diseña también un negocio gratuito".

La mayor parte de los entrevistados asegura que el nacimiento de estos clubs tampoco será la panacea que convierta a todos los usuarios en suscriptores, es decir, en lectores de pago. De momento -y según indican la mayoría de ellos- seguirán dependiendo de fuentes variadas de ingresos:

- quiosco;

- venta en bloque;

- publicidad en el formato tradicional;

- publicidad en internet;

- suscripciones online de pago.

Las diferencias existentes hasta el momento en todos los modelos españoles no son muy significativas y están todavía a cierta distancia de los modelos latinoamericanos, tanto en desarrollos tecnológicos (sistemas de geolocalización), como en la profundidad de las promociones, la calidad de algunos socios y establecimientos (American Express) y la comprobación del ahorro real.

Hemos podido constatar que la industria de los diarios es cada vez más una plataforma tecnológica -al igual que otras industrias culturales-, que crea un output o contenido consumido de acuerdo con las pautas propias del medio. Y los directivos más conscientes coinciden también en que la mejor estrategia de fidelización es el big data: "afinar tu producto cada vez más en función de lo que los clientes demanden".

En el fondo, lo que está sucediendo en la industria de los media es un proceso de transformación digital, que ya han conocido o están conociendo otros sectores de actividad: desde la banca hasta la automoción. Parece claro -en opinión de los directores generales con mayor perfil tecnológico- que las ventajas competitivas de las que gozaba la prensa en el pasado han desaparecido y ya no es posible mantener las economías de escala de antaño. El mercado dual basado en la publicidad ofrece síntomas de agotamiento, y es preciso entrar en sectores desconocidos bajo un entorno multiplataforma. Sin duda, un nuevo desafío para una industria con bastantes resabios anteriores y que está sufriendo una transformación muy similar a la que viene experimentando la televisión generalista, puesto que sobre ella comienzan también a converger tres industrias: audiovisual, telecomunicaciones e informática.

Los affinity clubs han demostrado en los países en los que se han implantado que han tenido éxito económico -como hemos comprobado en Latinoamérica-, al igual que el cobranding (caso de El periódico de Catalunya con el club de cultura TR3SC o del diario El mercurio con la tarjeta American Express), puesto que pueden ofrecer a sus socios importantes beneficios por el coste de la suscripción. Y aunque parece claro que es necesario disponer de una mínima masa

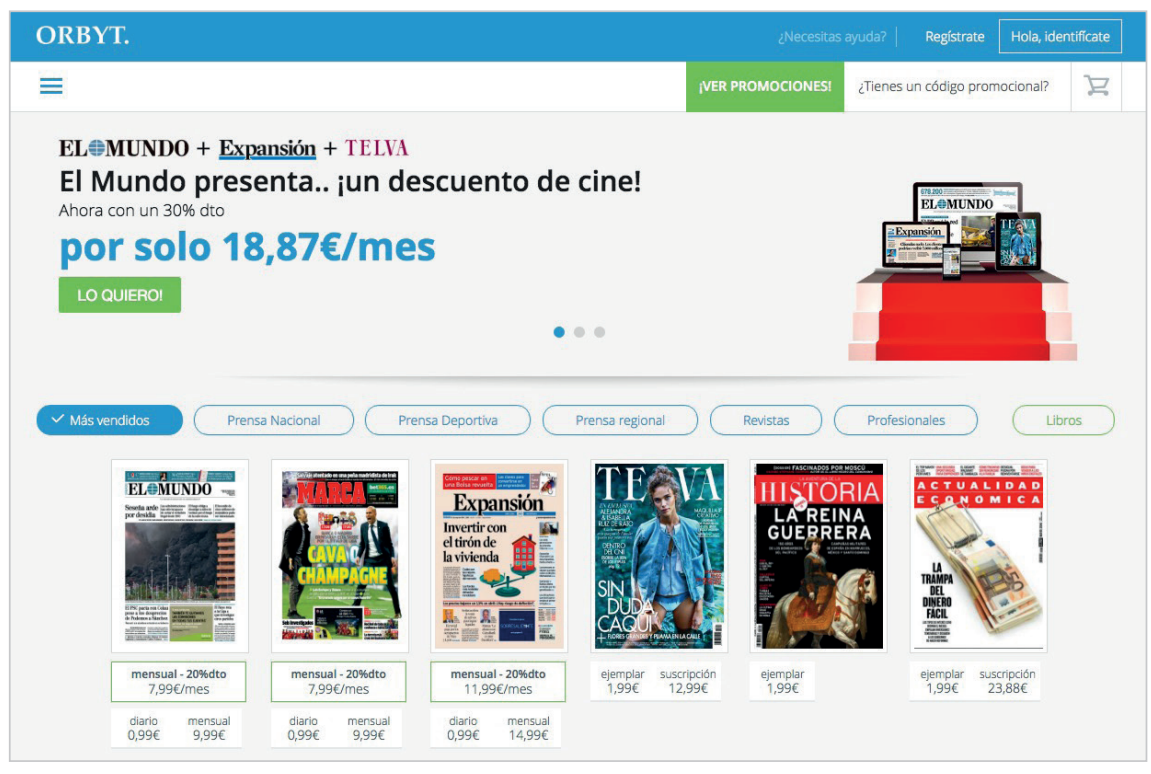

http://quiosco.orbyt.es 


\section{elPeriódico}

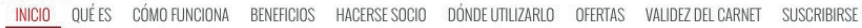

\section{BIENVENIDO AL CLUB DEL SUSCRIPTOR TR3SC}

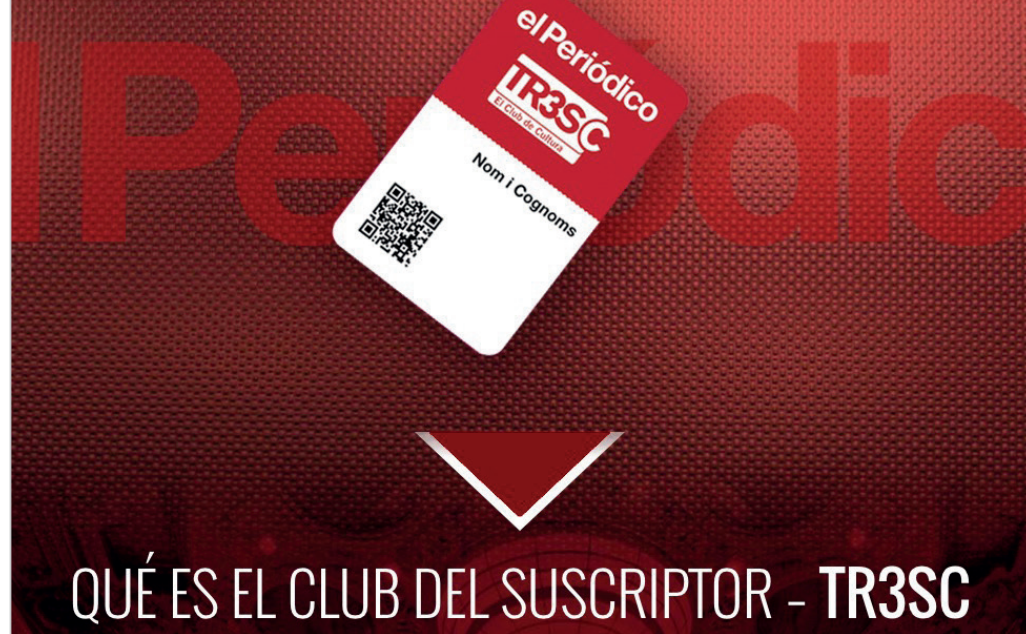

El Club del suscriptor - TR3SC es un colectivo de socios que disfrutan de numerosas ventajas en el ámbito del

ocio y la cultura.

Ser suscriptor de EL PERIÓDICO da derecho a pertenecer a este Club *, que ofrece descuentos permanentes en la taquilla de muchos locales catalanes y propuestas únicas para los socios.

http://especiales.elperiodico.com/suscriptores/club-suscriptor-tr3sc

crítica empresarial para poner en marcha clubs de estas características, algunos medios de prensa regional -caso de Última hora Mallorca (Grupo Serra)- también han sido capaces de llevar a cabo proyectos similares.

http://ultimahora.es/mallorca.html

Lo que está sucediendo en la industria de los medios es un proceso de transformación digital que ya han conocido otros sectores de actividad

Hasta el momento no existen datos que permitan constatar si estas iniciativas promovidas por los grandes grupos de comunicación, fomentarán o no una mayor vinculación de sus lectores, además de lograr mayores ingresos que compensen la pérdida de lectores en el entorno convencional y el descenso de la publicidad. Del mismo modo, resulta difícil saber también -según los entrevistados- si estas estrategias acabarán por estimular en el mercado español una mayor vinculación y fidelización de los clientes de prensa, así como una mayor generación de ingresos.

Ahora bien, ninguno de ellos ponía en duda que en un futuro cercano la principal fuente de ingresos de los diarios debería provenir de las aportaciones de los suscriptores, y que en el momento actual los clubs de suscriptores son la apuesta de futuro más acertada.

\section{Nota}

Este artículo es el resultado de la segunda fase del proyecto de investigación sobre financiación social de los media, de la UNIR y el Instituto para la Innovación Periodística (2IP), cuya primera parte culminó en el año 2014.

\section{Bibliografía}

AEDE (2015). Libro blanco de la prensa diaria. Madrid: Asociación de Editores de Diarios Españoles. ISBN: 9788460837954

Alfaro-Faus, Manuel (coord.) (2004). Temas claves en marketing relacional. Madrid: McGraw-Hill. ISBN: 8448142365

Anderson, Chris (2009). Free: The future of a radical price. Nueva York: Hyperion. ISBN: 9781401322908

Astigarraga, Eneko (2002). El método Delphi. Universidad de Deusto.

http://www.prospectiva.eu/zaharra/Metodo_ delphi.pdf

Bleyen, Valérie-Anne; Van-Hove, Leo (2007). "Western European newspapers and their online revenue models". First Monday, v. 12 , n. 12.

http://firstmonday.org/article/view/2014/1899

Cagé, Julia (2016). Salvar los medios de comunicación. Barcelona: Anagrama. ISBN: 9788433963963

Casero-Ripollés, Andreu (2010). "Prensa en internet: nuevos modelos de negocio en el escenario de la convergencia". El profesional de la información, v. 19, n. 6, pp. 595-601. http://recyt.fecyt.es/index.php/EPI/article/view/epi.2010. nov.05

http://dx.doi.org/10.3145/epi.2010.nov05

Chyi, Hsiang-Iris (2005). "Willingness to pay for online news: an empirical study on the viability of the subscription model". Journal of media economics, v. 18, n. 2, pp. 131-142. https://gjchatalas.files.wordpress.com/2009/10/willingness_to_pay_online_news.pdf http://dx.doi.org/10.1207/s15327736me1802_4

Díaz-Nosty, Bernardo (2013). La prensa en el nuevo ecosistema informativo. Que paren las rotativas. Barcelona: Fundación Telefónica/Ariel. ISBN: 9788408112969 http://boletines.prisadigital.com/la_prensa_en_el_nuevo_ ecosistema_informativo.pdf

García-Santamaría, José-Vicente (ed.) (2014). El negocio de la prensa digital. Madrid: UNIR. ISBN: 9788416125258 http://www.2ip.es/wp-content/uploads/2014/08/ELNEGOCIO-DE-LA-PRENSA-DIGITAL1.pdf

García-Santamaría, José Vicente; Clemente-Fernández, María-Dolores; López-Aboal, María (2013). "La organización de las redacciones en los nuevos diarios digitales españoles y su relación con los nuevos modelos de negocio". Textual \& visual media, n. 6, pp. 141-160. 
http://textualvisualmedia.com/images/revistas/06/articulos/ La\%20organizacion.pdf

Giesen, Edward; Riddleberger, Eric; Christner, Richard; Bell, Ragna (2010). "When and how to innovate your business model". Strategy \& leadership, v. 38, n. 4, pp. 17-26. http://dx.doi.org/10.1108/10878571011059700

Gómez-Borrero, Pilar (2015). Los clubs de suscriptores. ¿Tabla de salvación para la prensa? Instituto para la Innovación Periodística 2IP.

http://www.2ip.es/wp-content/uploads/2015/02/LOSCLUBS-DE-SUSCRIPTORES.pdf

Herbert, Jack; Thurman, Neil (2007). "Paid content strategies for news websites: An empirical study of British newspapers' online business models". Journalism practice, v. 1, n. 2, pp. 208-226.

http://openaccess.city.ac.uk/118

http://dx.doi.org/10.1080/17512780701275523

Herrero-Crespo, Ángel; Rodríguez-del-Bosque, Ignacio; Pérez-Ruiz, Andrea (2009). “Tarjetas de fidelización en el comercio minorista. Una herramienta de marketing relacional". Distribución y consumo, mayo-junio, pp. 50-63.

http://www.mercasa.es/files/multimedios/pag_050-063_ tarjetas.pdf

Leiva, Ricardo (2009). "El precio justo: Estrategias para cobrar por las noticias online". Cuadernos de información, julio-diciembre, n. 25, pp. 39-50.

http://cuadernos.info/index.php/CDI/article/view/45/42

Niles, Robert (2010). "There is no new revenue model for journalism". OJR: The online journalism review.

http://www.ojr.org/p1812

Palacios-Preciado, Mariana; Duque-Oliva, Edison-Jair (2011). "Modelos de negocio: propuesta de un marco conceptual para centros de productividad". Administración \& desarrollo, v. 53, n. 19, pp. 23-34.

https://dialnet.unirioja.es/servlet/articulo?codigo $=3776508$

Pérez-Bahón, Félix; Clemente-Fernández, María-Dolores; López-Aboal, María (2015). "El crowdfunding periodístico transforma el escenario mediático en España". En: Rodríguez-Rodríguez, Jorge-Miguel. Repensar los valores clásicos del periodismo. El desafío de una profesión enred@da. Actas del XXI Congreso Internacional de la SEP. Zaragoza: Universidad San Jorge, pp. 920-934. ISBN: 9788460831037
Picard, Robert G. (2002). The economics and financing of media companies. New York: Fordham University Press. ISBN: 9780823232574

Poulet, Bernard (2011). La fin des journaux et l'avenir de I'information. Paris: Gallimard. ISBN: 9782070441402

Rodríguez-Infiesta, Víctor (2008). "Suscriptores y suscripciones periodísticas en la España de principios del siglo XX. Apuntes desde una perspectiva asturiana". Hispania. Revista española de historia, v. LXVIII, n. 230, pp. 761-786.

http://dx.doi.org/10.3989/hispania.2008.v68.i230.93

Ruiz-Olabuenaga, José-Ignacio (2008). Metodología de la investigación cualitativa. Bilbao: Universidad de Deusto. ISBN: 9788474854237

Salaverría, Ramón (2010). "Estructura de la convergencia”. En: López-García, Xosé; Pereira-Fariña, Xosé (eds.). Convergencia digital. Reconfiguración de los medios de comunicación en España. Santiago de Compostela: Servicio Editorial de la Universidad de Santiago de Compostela, pp. 27-40. ISBN: 9788498873795

Sirkkunen, Esa; Cook, Clare (eds.) (2012). Chasing sustainability on the net. International research on 69 journalistic pure players and their business models. Tampere: Juvenes Print. ISBN: 9789514489679

https://tampub.uta.fi/bitstream/handle/10024/66378/ chasing_sustainability_on_the_net_2012.pdf?sequence $=1$

Taylor, Steven J.; Bogdan, Robert (1987). Introducción a los métodos cualitativos de investigación. Barcelona: Paidós, ISBN: 9788475098166 http://goo.gl/dtBPvQ

Vara-Miguel, Alfonso (2014): "Tendencias en el cobro por contenidos digitales". En: AEDE. Libro blanco de la prensa diaria. Madrid: Asociación de Editores de Diarios Españoles, pp. 179-190. ISBN: 9788460837954

Varela, Juan (2011). "Perspectivas y retos. Periodismo mutante". Telos, n. 86, pp. 107-110.

https://telos.fundaciontelefonica.com/seccion=1268\&idioma=es_ ES\&id $=2011012610500001$ \&activo $=6$. do

Zott, Christoph; Amit, Raphael (2009). "Innovación del modelo de negocio: creación de valor en tiempos de cambio". Universia business review, n. 23, pp. 108-121. http://www.redalyc.org/articulo.oa?id=43312282008 